\title{
High fidelity dissipation engineering using parametric interactions
}

\author{
E. Doucet, ${ }^{1, *}$ F. Reiter, ${ }^{2}$ L. Ranzani, ${ }^{3}$ and A. Kamal ${ }^{1,4, \dagger}$ \\ ${ }^{1}$ Department of Physics and Applied Physics, University of Massachusetts, Lowell, Massachusetts 01854, USA \\ ${ }^{2}$ Harvard University, Department of Physics, 17 Oxford St, Cambridge, Massachusetts 02138, USA \\ ${ }^{3}$ Raytheon BBN Technologies, Cambridge, Massachusetts 02138, USA \\ ${ }^{4}$ Research Laboratory of Electronics, Massachusetts Institute of Technology, Cambridge, Massachusetts 02139, USA
}

(Received 15 October 2018; revised manuscript received 29 March 2020; accepted 3 June 2020; published 19 June 2020)

\begin{abstract}
Quantum reservoir engineering provides a versatile framework for quantum state preparation and control, with improved robustness to decoherence. However, established methods for dissipative state preparation typically rely on resolving resonances, limiting the target state fidelity due to a competition between the stabilization mechanism and uncontrolled dissipation. We propose a new framework for engineering dissipation that combines the advantages of static dispersive couplings with strong parametric driving and show how it can realize high fidelity and fast entanglement stabilization devoid of such constraints. In addition, the phase sensitivity of parametric couplings allows arbitrary state preparation and continuous control of the stabilized state within a fixed parity manifold. The proposed protocol is readily accessible with the state-of-the-art superconducting qubit technology and holds promise for fast preparation of large entangled resource states.
\end{abstract}

DOI: 10.1103/PhysRevResearch.2.023370

\section{INTRODUCTION}

Quantum state preparation and preservation are the cornerstones of any quantum information platform. Standard methods of state preparation involve a set of unitary operations (or gates) on individual and multiple qubits to achieve a desired entangled state of the system. Such methods typically require multiple tightly synchronized pulse sequences and ancilla qubits, and complex algorithms to avoid unwanted interactions and contain the quantum information within the desired subspace. The prepared state also remains sensitive to environmental decoherence, which reduces the pure quantum state into a classical mixture and ultimately limits the power to harness quantum effects. Recent years have seen an emergence of an alternative approach embracing the environment instead of hiding it $[1,2]$. The basic idea relies on engineering suitable interactions with the environment that steer the reduced system towards a desired target state $[3,4]$. Besides engineering states inherently robust to dissipation, such dissipative state preparation precludes the need for any active control of the system and is relatively immune to initialization errors. Also, certain quantum operations become feasible only through dissipation; for instance, nonlocal interactions are essential for the stabilization of multi-partite entangled states such as the three-qubit GHZ state $[5,6]$. This has led to interest

\footnotetext{
*emery_doucet@student.uml.edu

†archana_kamal@uml.edu
}

Published by the American Physical Society under the terms of the Creative Commons Attribution 4.0 International license. Further distribution of this work must maintain attribution to the author(s) and the published article's title, journal citation, and DOI. in dissipative preparation techniques being adopted in a gamut of quantum information platforms such as cavity QED $[7,8]$, trapped ions [9-11], superconducting qubits [12,13], Rydberg atoms [14,15], atomic ensembles [16], and NV centers [17]. Though they offer a powerful approach to protecting quantum information, almost all dissipative state preparation schemes exhibit a tradeoff between target fidelity and stabilization time; thus accurate state preparation necessarily requires long times.

In this paper, we present a novel paradigm for engineering dissipation using number-selective, strong parametric driving free of such constraints. The framework of parametrically engineered dissipation proposed here constitutes a significant addition to the parametric quantum toolbox, which is being wielded in a wide variety of applications, such as entangling gates [18-20], dynamical correction of qubit errors [21], nonreciprocal scattering [22], and synthetic magnetic fields [23], holonomic gates for continuous-variable quantum information [24] and even quantum annealing [25].

We demonstrate that this platform enables robust, high fidelity entanglement generation and control in a circuit-QED setup [26] by describing a scheme for stabilization of Bell states in a dispersively and parametrically coupled qubitresonator system. In contrast to usual dissipation-engineering schemes which rely on resonant driving, our scheme exhibits concurrent scaling between the fidelity and speed of stabilization protocol achieving preparation of Bell-states with fidelities in excess of $99 \%$ in a few $100 \mathrm{~ns}$. It also leverages several technical and operational advantages offered by parametric driving, such as easy implementation relying on continuous-wave $(\mathrm{CW})$ drives alone, strong coupling even in the nonresonant regimes unlike the strong-dispersive circuitQED [27], and in situ tunability of coupling phases. In particular, the phase tunability of the couplings translates directly 


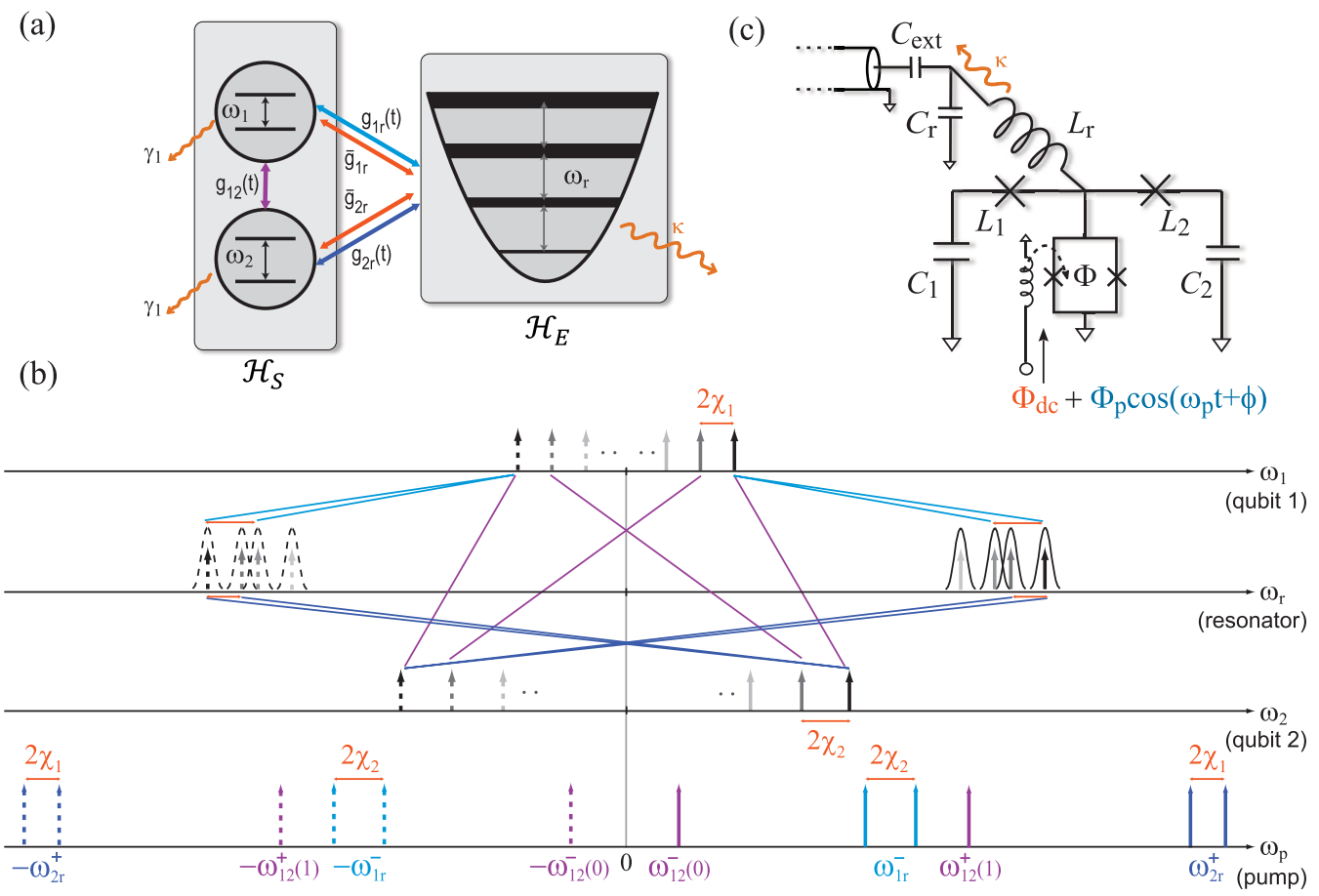

FIG. 1. (a) Block diagram of a system of qubits with pairwise parametric and dispersive couplings to a resonator acting as an engineered environment. (b) Frequency landscape showing the various parametric mixing processes between the qubits and the resonator, and the respective pumps. Solid and dashed vertical arrows indicate the lines in qubit-resonator spectra and their conjugates, respectively. The dispersive shifts due to static couplings $\left(\bar{g}_{j r}\right)$ are indicated in red arrows. The parametric couplings $\left(g_{12}(t), g_{j r}(t)\right)$ are shown as lines mixing different frequencies across qubit-resonator axes; the processes with the lines crossing zero represent two-photon excitation processes, while those confined to mixing between positive (or negative) frequencies represent excitation-preserving hopping. Parametrically driven number-sensitive qubit-qubit interactions are shown with purple lines, while qubit-resonator interactions are shown with blue lines. (c) Circuit implementation of the Bell state stabilization protocol consisting of two superconducting transmon qubits and a microwave resonator coupled in a T-configuration to a dc-SQUID, driven via an external flux line. The linear inductance of the SQUID corresponding to the static flux bias $\left(\Phi_{\mathrm{dc}}\right)$ mediates a dispersive coupling, while the fast flux modulation $\left(\Phi_{p}\right)$ implements parametric driving through the SQUID nonlinearity.

to in situ control of the target state within a fixed parity manifold.

\section{PARAMETRICALLY ENGINEERED DISSIPATION}

Our proposed framework for quantum state engineering consists of a system of qubits coupled to reservoir modes using a combination of pairwise parametric and dispersive couplings. Figure 1(a) schematically represents a two-qubit Bell state stabilization scheme within this framework. The system is described by a generic Hamiltonian of the form

$$
\mathcal{H}=\omega_{r} a^{\dagger} a+\sum_{j=1}^{2}\left(\frac{\omega_{j}}{2} Z_{j}+\tilde{g}_{j r}(t) X_{j} X_{r}\right)+\tilde{g}_{12}(t) X_{1} X_{2},
$$

where $X_{j}=\sigma_{j}+\sigma_{j}^{\dagger} X_{r}=\left(a+a^{\dagger}\right)$. In addition to the usual static dispersive coupling, parametric modulation via a continuous-wave pump is employed such that

$$
\begin{aligned}
& \tilde{g}_{12}(t)=g_{12}^{+} \cos \left(\omega_{12}^{+} t+\phi_{12}^{+}\right)+g_{12}^{-} \cos \left(\omega_{12}^{-} t+\phi_{12}^{-}\right), \\
& \tilde{g}_{j r}(t)=\bar{g}_{j r}+g_{j r}^{ \pm} \cos \left(\omega_{j r}^{ \pm} t+\phi_{j r}^{ \pm}\right) .
\end{aligned}
$$

Different coupling terms can be activated in the systemenvironment Hamiltonian by choosing the modulation frequencies as either the sum $\omega_{j r}^{+}=\omega_{j}+\omega_{r}$ or difference $\omega_{j r}^{-}=\omega_{j}-\omega_{r}$ of the resonance frequencies of the qubits and resonator. In addition leveraging the splitting of the qubit spectrum due to strong dispersive couplings, selective qubitqubit couplings can be activated depending on the number of excitations in the resonator. The scheme is designed such that the qubit-qubit drives couple states of the same parity, while the qubit-resonator drives mix the different parity manifolds. In conjunction with resonator decay [denoted $\kappa$ in Fig. 1(a)], this pumping scheme is capable of stabilizing any maximally entangled two-qubit state of a fixed parity depending on the choice of drive frequencies.

For instance, Fig. 1(b) shows the pump frequencies

$$
\begin{aligned}
\omega_{12}^{+}(n) & =\omega_{1}+\omega_{2}-2 n\left(\chi_{1}+\chi_{2}\right), \\
\omega_{12}^{-}(n) & =\omega_{1}-\omega_{2}-2 n\left(\chi_{1}-\chi_{2}\right), \\
\omega_{1 r}^{-} & =\omega_{1}-\omega_{r}-\chi_{1} \pm \chi_{2}, \\
\omega_{2 r}^{+} & =\omega_{2}+\omega_{r}-\chi_{2} \pm \chi_{1} .
\end{aligned}
$$

for $n=1$ desired for preparing even-parity Bell states. For this choice of pumps the two-photon excitation of the qubits is active only when there is one-photon in the resonator, while qubit-qubit hopping is activated when the resonator is in its ground state. This is evident on moving into a rotating frame defined with respect to the number-dependent frequencies, 


$$
\begin{aligned}
& \omega_{j}(n)=\omega_{j}-2 \chi_{j r} a^{\dagger} a, \\
& U(n)=\exp \left[-i\left(\frac{\omega_{1}(n)}{2} Z_{1}+\frac{\omega_{2}(n)}{2} Z_{2}+\omega_{r} a^{\dagger} a\right) t\right],
\end{aligned}
$$

which leads to an effective Hamiltonian of the form (for details, see Appendix A),

$$
\begin{aligned}
\mathcal{H}_{\text {eff }}^{\text {even }}= & g_{12}^{-} e^{i \phi_{12}^{-}}\left(1-\left\langle a^{\dagger} a\right\rangle\right) \sigma_{1} \sigma_{2}^{\dagger}+g_{12}^{+} e^{i \phi_{12}^{+}}\left\langle a^{\dagger} a\right\rangle \sigma_{1} \sigma_{2} \\
& +\left(g_{1 r}^{-} e^{i \phi_{1 r}^{-}} \sigma_{1}+g_{2 r}^{+} e^{i \phi_{2 r}^{+}} \sigma_{2}^{\dagger}\right) a^{\dagger}+\text { H.c. }
\end{aligned}
$$

in the subspace truncated at lowest two levels of the resonator. Similarly, for preparing odd-parity states, choosing modulation frequencies $\omega_{12}^{+}(0), \omega_{12}^{-}(1), \omega_{1 r}^{-}$, and $\omega_{2 r}^{+}$leads to an interaction of the form

$$
\begin{aligned}
\mathcal{H}_{\mathrm{eff}}^{\mathrm{odd}}= & g_{12}^{+} e^{i \phi_{12}^{+}}\left(1-\left\langle a^{\dagger} a\right\rangle\right) \sigma_{1} \sigma_{2}+g_{12}^{-} e^{i \phi_{12}^{-}}\left\langle a^{\dagger} a\right\rangle \sigma_{1} \sigma_{2}^{\dagger} \\
& +\left(g_{1 r}^{+} e^{i \phi_{1 r}^{+}} \sigma_{1}^{\dagger}+g_{2 r}^{+} e^{i \phi_{2 r}^{+}} \sigma_{2}^{\dagger}\right) a^{\dagger}+\text { H.c. }
\end{aligned}
$$

It is worthwhile to highlight here the features of the Hamiltonians presented in Eqs. (5) and (6) that distinguish them from typical state preparation protocols. (i) As described above, the form of each of the coupling terms is uniquely set by the choice of frequency of the pump mediating the coupling. (ii) Additionally, the dispersive qubit-resonator couplings lead to a selective activation of the qubit-qubit couplings contingent on the photon occupation in the resonator. (iii) By virtue of the couplings being parametric, their strengths and phases are tunable through the amplitudes and phases of the pumps. This freedom of coupling parameters, combined with a decay on the resonator, provides a convenient method for realizing different parametrically engineered collapse operators $c_{p}$. Though the choice of $\omega_{j k}$ and $\phi_{j k}$, we engineer the target state to be a null ket (dark state) of both the system Hamiltonian and jump operators, ensuring that the target state is the unique fixed point (steady-state) of the system dynamics [1].

Figure 1(c) shows a circuit-QED implementation of our proposed implementation, consisting of two superconducting transmon qubits and a microwave LC resonator coupled via a SQUID-based tunable inductance. The microwave resonator coupled to an external superconducting transmission line via a coupling capacitor acts as an engineered bath to the qubits. For a discussion of the static and parametric coupling rates accessible with our proposed implementation, as well as a discussion of the effect of the coupler design on qubit decoherence, see Appendix B.

\section{A. Stabilization mechanism}

We now describe how the above functionalities may be exploited to dissipatively stabilize any maximally entangled two-qubit state. The generic coupling map in Fig. 2 describes the stabilization mechanism for all four Bell states, with an appropriate relabeling of states and optimal choice of pump phases as stated in Table I. For the purposes of illustration, we describe the mechanism for the specific case of evenparity Bell state preparation, implemented using the effective interaction Hamiltonian of Eq. (5). Assume that the two qubits have been stabilized into $\left|\Phi_{-}\right\rangle=(|g g\rangle-|e e\rangle) / \sqrt{2}$. Under a bit flip, the parity of the state changes leading to a jump to

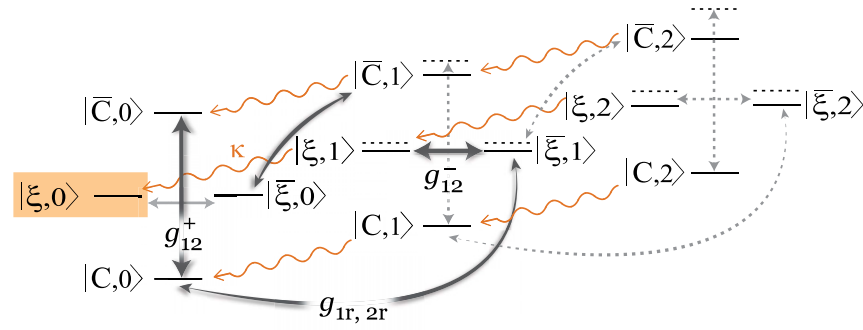

FIG. 2. Coupling diagram for two-qubit entanglement stabilization. Thick black arrows denote resonant interactions, while dotted gray arrows denote off-resonant couplings. Wavy arrows depict photon decay from the resonator. The target state (highlighted with the colored box) and the complementary state (phase-flipped but same parity) are denoted with Greek letters $|\xi\rangle,|\bar{\xi}\rangle$, respectively. The states in the opposite parity manifold are denoted with $|C\rangle,|\bar{C}\rangle$, respectively.

the odd parity manifold spanned by $\{|g e\rangle,|e g\rangle\} \otimes|0\rangle$. Each of these odd-parity states is coherently coupled to the even-parity state $\left|\Phi_{+}, 1\right\rangle$, either directly using the qubit-resonator drives (for $|e g\rangle$ ) or through a combination of qubit-qubit and qubitresonator drives (for $|g e\rangle$ ), while exciting the resonator. The remaining qubit-qubit drive $g_{12}^{-}$pumps the population from $\left|\Phi_{+}, 1\right\rangle$ to $\left|\Phi_{-}, 1\right\rangle$, which then decays down to the target state $\left|\Phi_{-}, 0\right\rangle$ as the resonator loses the photons at rate $\kappa$. Any population that enters the state $\left|\Phi_{-}, 0\right\rangle$ remains unaffected by the qubit-qubit drive and the qubit-resonator drive, since $\left|\Phi_{-}\right\rangle$is a dark state of both the qubit Hamiltonian and the engineered collapse operator $c_{p}=\sigma_{1}+\sigma_{2}^{\dagger}$. A phase flip on a qubit moves the population to $\left|\Phi_{+}\right\rangle$; this state strongly couples to the oddparity state $|g e, 1\rangle$ through the qubit-resonator interaction. The excitation of the resonator induces a dispersive shift of the qubit frequency making the $g_{12}^{+}$drive off-resonant for this state; hence the dominant exit channel is the resonator decay which brings back the population to odd-parity manifold with no photons in the resonator. This is then repumped to $\left|\Phi_{-}, 0\right\rangle$ as described for the case of qubit decay. It is worthwhile to note that the mechanism works for any arbitrary initial state including maximally mixed states of the two qubits.

\section{B. Continuous-wave coherent control}

The phases of the parametric couplings are determined by the phases of the pumps mediating the respective interactions. This provides a unique knob to implement rotations in the two-qubit stabilized subspace. For example, Table I shows that when stabilizing an even-parity state $\left(|g g\rangle-e^{i \psi}|e e\rangle\right) / \sqrt{2}$, varying the qubit-qubit and qubit-resonator coupling phases

TABLE I. State vectors, pump phases, and respective parametrically engineered collapse operators for arbitrary maximally entangled states of even or odd parity.

\begin{tabular}{lcccccl}
\hline \hline$\sqrt{2}|\xi\rangle$ & $|C\rangle$ & $\phi_{12}^{+}$ & $\phi_{12}^{-}$ & $\phi_{1 r}$ & $\phi_{2 r}$ & $c_{p}$ \\
\hline$|g g\rangle-e^{i \psi}|e e\rangle$ & $|e g\rangle$ & $\pi / 2-\psi$ & 0 & 0 & $\psi$ & $\sigma_{1}+e^{i \psi} \sigma_{2}^{\dagger}$ \\
$|g e\rangle-e^{i \psi}|e g\rangle$ & $|g g\rangle$ & 0 & $\pi / 2-\psi$ & 0 & $\psi$ & $\sigma_{1}+e^{i \psi} \sigma_{2}$ \\
\hline \hline
\end{tabular}


together as

$$
\psi=\phi_{2 r}=\pi / 2-\phi_{12}^{+},
$$

allows the target state to be continuously tuned.

\section{PERFORMANCE ANALYSIS}

The dynamics of the full qubits-resonator system can be described using a Liouvillian as $\dot{\rho}(t)=\mathcal{L} \rho(t)$, where

$$
\begin{aligned}
\mathcal{L}= & -i\left[\mathcal{H}_{\mathrm{eff}}, \bullet\right]+\frac{\kappa}{2} \mathcal{D}[a] \bullet \\
& +\sum_{j=1,2}\left(\frac{\gamma_{1}^{j}}{2} \mathcal{D}\left[\sigma_{j}\right] \bullet+\frac{\gamma_{\phi}^{j}}{2} \mathcal{D}\left[Z_{j}\right] \bullet\right)
\end{aligned}
$$

with $\mathcal{D}[o] \bullet=2 o \bullet o^{\dagger}-\left\{o^{\dagger} o, \bullet\right\}$ being the usual Lindblad superoperators describing Markovian decay dynamics for the two qubits and the resonator. Here $\kappa$ denotes the bare resonance linewidth of the resonator, while $\left(\gamma_{1}^{j}, \gamma_{\phi}^{j}\right)$ denote the qubit relaxation and dephasing rates respectively. We note that use of a local Markovian dissipator in Eq. (8), describing the dominant decay through the resonator acting as an engineered bath for qubits, is motivated by the dispersive circuit-QED regime considered here, $\gamma_{1}^{j} \ll \kappa, g_{j k} \ll$ $\Delta_{j r}$. The first inequality ensures that the density of states of the resonator appears flat (frequency-independent) over qubit response times, while the second inequality ensures that transitions between widely separated frequencies remain offresonant in the presence of interaction-induced energy-level splittings. While strong parametric driving introduces additional nonlocal channels of dissipation, the associated rates are sufficiently small such that their effect on the overall stabilization dynamics can be ignored, as shown in Appendix C. These corrections can become important in the presence of colored noise or ultrastrong qubit-resonator coupling [28]. In these scenarios, the rotating-wave approximation breaks down, leading to unphysical predictions [29]. In such cases, the master equation would need to be derived in the dressed basis of the qubit-resonator system in order to identify the correct frequency-dependent dissipation rates [30].

At any instant in time during the operation of the stabilization protocol, the error to the target state is

$$
\varepsilon(t)=1-\operatorname{Tr}\left[\rho(t) \mathbb{I}_{\mathrm{res}} \otimes|\xi\rangle\langle\xi|\right] .
$$

where $\rho(t)$ is the density operator describing the state of the full qubit-resonator system. This error can be decomposed into a static component $\left(\varepsilon_{\infty}\right)$ and a dynamical component $(\tilde{\varepsilon})$ as

$$
\varepsilon(t) \approx \varepsilon_{\infty}+\tilde{\varepsilon} \exp (-t / \tau),
$$

where the dynamical error decays exponentially to zero as $t \rightarrow \infty$ with some characteristic stabilization time $\tau$, leaving only the steady-state error $\varepsilon_{\infty}$. Both $\varepsilon_{\infty}$ and $\tau$ may be accurately determined directly from the Liouvillian as

$$
\begin{aligned}
\varepsilon_{\infty} & =1-\operatorname{Tr}\left(\rho_{s s} \mathbb{I}_{\text {res }} \otimes|\xi\rangle\langle\xi|\right), \\
\tau & =\Delta_{\mathcal{L}}^{-1}=-\operatorname{Re}\left[\lambda_{1}\right]^{-1},
\end{aligned}
$$

where $\rho_{s s}$ is the steady-state density matrix, defined by $\mathcal{L} \rho_{s s}=$ 0 , and $\lambda_{1}$ is the lowest lying nonzero eigenvalue of the Liouvil- lian [1]. We have confirmed that this method of calculating the performance metrics is in excellent agreement with numerical simulations of the master equation, with relative errors on the order of $10^{-6}$ for both $\varepsilon_{\infty}$ and $\tau$.

\section{A. Steady-state optimization}

Figure 3(a) shows the results of a multi-parameter optimization of the steady-state error calculated from the Liouvillian. Here, for simplicity of presentation, we have assumed symmetric qubit-qubit couplings $g_{12}^{ \pm}=g$ and qubit-resonator couplings $g_{j r}^{ \pm}=g_{r}$. As evident, a salient feature of our scheme is the simultaneous reduction in steady-state error, $\varepsilon_{\infty}$, and preparation time, $\tau$, scaling as $1 / g$ when $\kappa=2 g_{r}=(3 / 2) g$.

To understand this unique property, we perform an analytical calculation of the steady-state error and stabilization time using a Liouvillian truncated to include the lowest two resonator levels. Assuming that the mixing of different states (off-diagonal elements of $\rho_{s s}$ ) due to qubit decays can be ignored, as $\gamma \ll\left(g, g_{r}, \kappa\right)$, we can obtain expressions for $\varepsilon_{\infty}$ by performing expansion in the relevant small parameter $\gamma / \max \{\kappa, g\}$. To first order the steady-state error in the $\kappa$ dominated (or strong dissipation) and $g$-dominated (or weak dissipation) regimes is found to be

$$
\varepsilon_{\infty}= \begin{cases}12.6\left(\frac{\gamma_{1}}{\kappa}\right) ; & \kappa \gg g \\ 22.4\left(\frac{1}{\mathcal{C}}\right) ; & \kappa \ll g,\end{cases}
$$

where we have considered the optimal ratio between qubitqubit and qubit-resonator coherent couplings, $g_{r}=(3 / 4) g$. Here, $\mathcal{C}=\left(4 g^{2} / \kappa \gamma_{1}\right)$ denotes the cooperativity of the system. Minimizing the total error as the sum of two contributions leads to an optimality condition for the ratio $\kappa / g=(3 / 2)$, with the minimum error that scales inversely with parametric coupling strength $g$

$$
\varepsilon_{\infty}^{\min } \approx 16.8\left(\frac{\gamma_{1}}{g}\right) .
$$

This optimal ratio between dissipative and coherent couplings is in excellent agreement with that obtained using full numerical optimization, as shown in Fig. 3(b).

Detailed numerical studies also show that the stabilization fidelity is robust to imperfections. Fidelities in excess of $99 \%$ are achievable for as large as 50\% deviations from optimal values of $g_{r}$ or $\kappa$, or for asymmetries in the qubit-qubit couplings $g_{12}^{+} \neq g_{12}^{-}$. We find that the performance of the scheme however, is more sensitive to asymmetries in the parametric qubit-resonator couplings $g_{1 r} \neq g_{2 r}$ as this introduces a spurious coherent coupling out of the target state. Such spurious couplings may also arise due to imbalance in qubit detunings; specifically, maximum steady-state fidelities are achieved for odd-parity states when $\delta_{1}=\delta_{2}$, and for even-parity states when $\delta_{1}=-\delta_{2}$. For a detailed discussion of the robustness of the scheme, see Appendix D.

An analytical calculation of stabilization time $\tau$ is more complicated since it is derived from the spectral gap of the Liouvillian, which requires a large matrix inversion. For instance, even for a rather conservative system comprising 

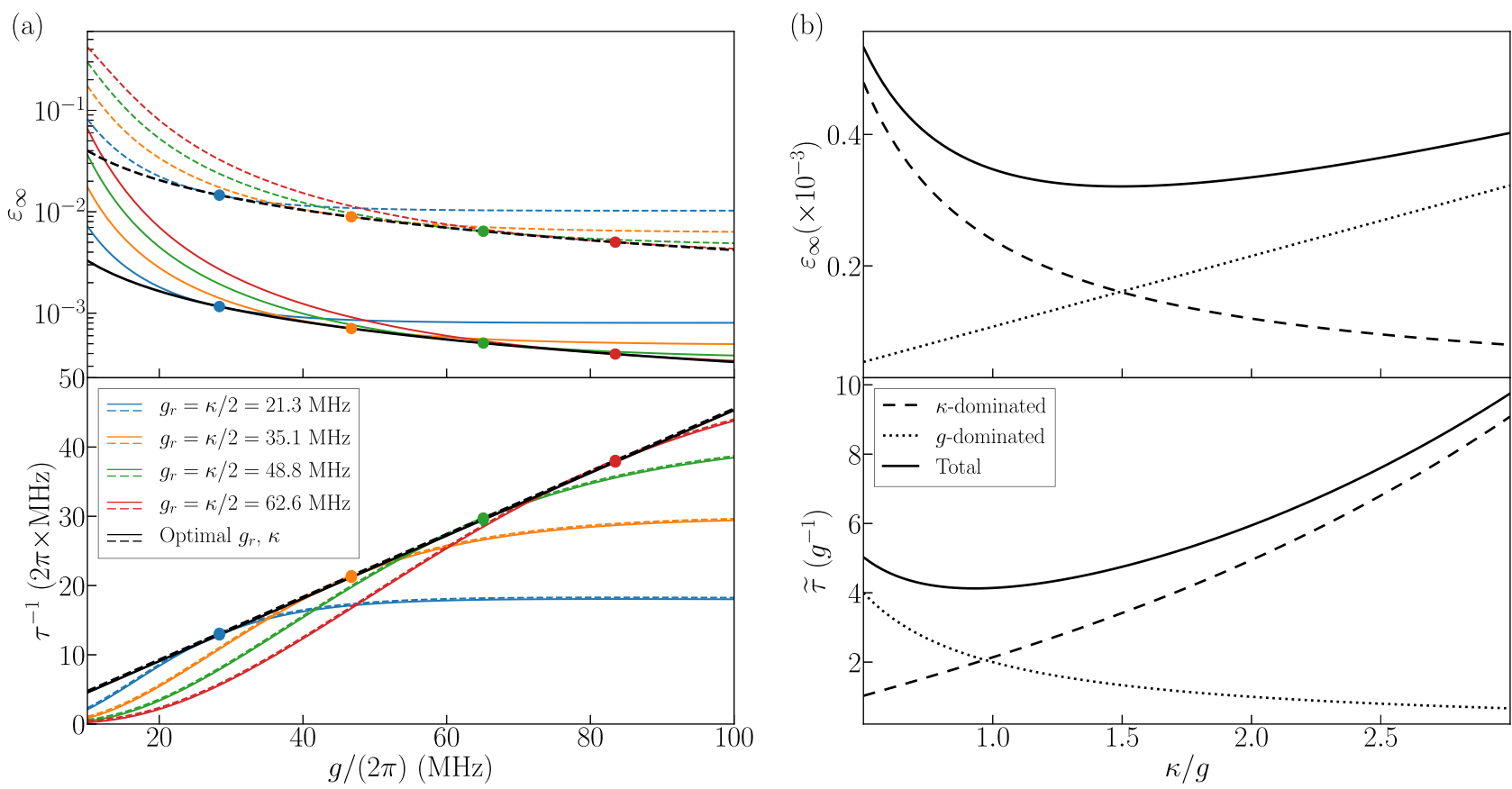

FIG. 3. (a) Numerical estimates of the steady-state error and convergence rate for the scheme depicted in Fig. 2, calculated as a function of the parametric coupling strength $g$. The black lines show the results for optimal parameters, $\kappa=2 g_{r}=(3 / 2) g$, with each of the colored dots indicating the value of $g$ for which $\left(g_{r}, \kappa\right)$ are optimal. The solid lines are calculated for $T_{1}=100 \mu \mathrm{s}, T_{2}=200 \mu \mathrm{s}$ ("best case"), while the dashed lines are calculated for $T_{1}=10 \mu \mathrm{s}, T_{2}=10 \mu \mathrm{s}$ ("worst case"). The qubit decoherence rates affect the target fidelity, but not the convergence time to target state. (b) Analytical estimates of the total steady-state error, denoted by $\varepsilon_{\infty}$, and lifetime of the state $|C, 0\rangle$ decaying into $|\xi, 0\rangle$, denoted by $\widetilde{\tau}$. Each plot also shows the result calculated in the limit $\kappa \gg g$ ( $\kappa$-dominated) and in the limit $\kappa \ll g$ ( $g$-dominated), for a fixed ratio $g_{r} / g=3 / 4$.

two qubits and a resonator truncated at two levels, an exact calculation involves diagonalization of a $64 \times 64$ Liouvillian. Nonetheless, an analytical estimate and qualitative scaling with parameters can be obtained by identifying a minimal decoupled subspace including the target state, and selfconsistently solving for the populations in different states spanning the subspace. The states $\{|C, 0\rangle,|\bar{\xi}, 1\rangle,|\xi, 1\rangle,|\xi, 0\rangle\}$ form such a subspace as seen in Fig. 2 , in which we can write the rate of preparation of the target state $|\xi, 0\rangle$ as

$$
\dot{P}_{|\xi, 0\rangle}(t)=\Gamma_{C, 0} P_{|C, 0\rangle}+\Gamma_{\bar{\xi}, 1} P_{|\bar{\xi}, 1\rangle}+\Gamma_{\xi, 1} P_{|\xi, 1\rangle},
$$

where $P_{|\psi\rangle}$ is the population in state $|\psi\rangle$ and $\Gamma_{|\psi\rangle}$ is the decay rate of $|\psi\rangle$ into the target state $|\xi, 0\rangle$. Following the same procedure as for the calculation of steady-state error, we obtain the expressions for $\Gamma$ in the decay- and couplingdominated regimes as

$$
\Gamma_{C, 0}=\left\{\begin{array}{cl}
0.49\left(\frac{g^{2}}{\kappa}\right) ; & \kappa \gg g \\
\frac{\kappa}{2} ; & \kappa \ll g,
\end{array}\right.
$$

calculated assuming $g_{r}=(3 / 4) g$. The above expressions lead to a net preparation rate,

$$
\begin{aligned}
\tilde{\tau}^{-1} & =\Gamma_{\mathrm{eff}}=\left(\left.\Gamma_{C, 0}^{-1}\right|_{\kappa \ll g}+\left.\Gamma_{C, 0}^{-1}\right|_{\kappa \gg g}\right)^{-1} \\
& \approx \frac{\kappa g^{2}}{2\left(g^{2}+\kappa^{2}\right)} .
\end{aligned}
$$

The analytical estimate of preparation rate in Eq. (16) serves as an upper bound for the exact result obtained from the Liouvillian gap, i.e. $\Gamma_{\text {eff }} \geqslant \operatorname{Re}\left[\Delta_{\mathcal{L}}\right]$. This is because the total convergence rate is determined by a series of processes that shuffle excitations between multiple decoupled subspaces. It is also worth noting that, to leading order, qubit decays do not affect the convergence time of the scheme as also confirmed by numerical optimization.

If we apply the optimality condition $\kappa / g=(3 / 2)$ as found numerically and in the above analysis of the steady-state error, we find that the preparation time $\tau$ scales with $1 / g$, in tandem with $\varepsilon_{\infty}$ as expected from our numerical analysis. This concurrent scaling may be understood by noting that in the absence of qubit decoherence $\left(\gamma_{1}=0\right), \varepsilon_{\infty}=0$ exactly, independent of $g$ as expected from Eq. (13) and verified numerically. Local dissipation on the qubits introduces an error channel out of the target state $|\xi\rangle$. The population in the target state will be static when this outflow due to decoherence is exactly balanced by the inflow due to the engineered dissipation. Thus, if the engineered dissipation rate is increased (reducing the stabilization time by increasing $g$ ), the steadystate error decreases consistently with the reduced ratio of the decoherence and preparation rates and converges to $\varepsilon_{\infty} \sim$ $\gamma_{1} / \Gamma_{\text {eff }}$. Such simultaneous reduction of stabilization error and preparation time is a generic property of any dissipative stabilization scheme where the target state is the unique dark state of both the system Hamiltonian and jump operators. In usual dissipative protocols, since the target state is an approximate dark state of the drive Hamiltonian, increasing the drive 


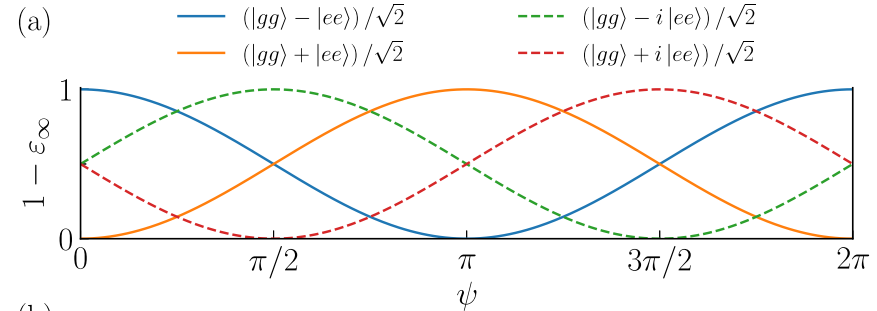

(b)

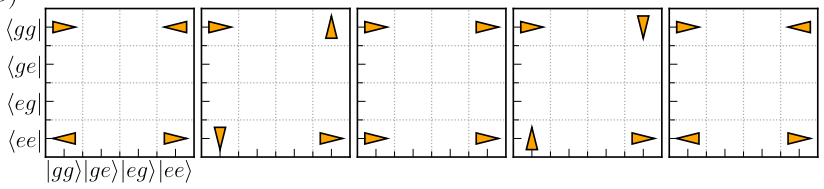

FIG. 4. Continuous rotations in the stabilized manifold of maximally entangled even-parity states. (a) Steady-state fidelities calculated from the Liouvillian, as a function of the target state phase $\psi$. (b) Tomograms of $\rho_{s s}$ for $\psi=k \pi / 2$. The opacity and direction of the arrows indicates the magnitude and phase of the matrix elements respectively. All calculations were done for $T_{1}=100 \mu \mathrm{s}, T_{2}=200 \mu \mathrm{s}, g=2 \pi \times 50 \mathrm{MHz}$, and with optimal values for the other parameters $\kappa^{\mathrm{opt}}=2 g_{r}^{\mathrm{opt}}=(3 / 2) g$.

strength introduces additional drive-dependent error channels and forces a compromise between fidelity and speed. Thus typical schemes operate in either the $\kappa$-dominated regime [31] [decreasing $\varepsilon_{\infty}$, increasing $\tau$; dashed-black curves in Fig. 3(b)] or the $g$-dominated regime [32] [increasing $\varepsilon_{\infty}$, decreasing $\tau$; dotted-black curves in Fig. 3(b)], and are constrained to maintain the product $\varepsilon_{\infty} \tau$ constant. We note that the preceding discussions of the performance of the scheme do not make any assumption about the target state $|\xi\rangle$ as defined in Table I. Hence the steady-state error and preparation time are independent of the choice of $|\xi\rangle$, as selected by the relative phases of the unitary and dissipative parametric couplings. Figure 4 demonstrates this by showing that stabilization of any maximally entangled state of even parity yields the same steady-state error. Further, such tuning of the target state preserves the purity and population in each parity manifold.

\section{B. Counter-rotating terms}

The analysis in previous sections considers only the resonant Hamiltonian obtained under the rotating wave approximation. Figure 5 shows the performance metrics for evenparity Bell state stabilization in the presence of the dominant off-resonant terms, not captured by Eqs. (5) and (6). The essential physics of these terms can be captured by including a $\chi$-dependent coherent leakage out of the target state in the zero-photon manifold,

$$
\mathcal{H}_{\mathrm{CR}} \approx g e^{i \phi_{12}^{ \pm}}\left(e^{-i 2 \Omega_{\chi} t}|\bar{\xi}, 0\rangle\langle\xi, 0|\right)+\text { H.c. },
$$

where $\Omega_{\chi}=\chi_{1} \pm \chi_{2}$. The phase on the prefactor and the sign in the definition of $\Omega_{\chi}$ is determined by the pump frequencies; when preparing an even parity Bell state + is selected, and for odd.

As is clear from Fig. 5, the scaling of $\varepsilon_{\infty}$ in the presence of $\chi$-dependent leakage qualitatively differs from that presented in Fig. 3 which was calculated using the resonant Hamiltonian [Eq. (5)]. This is because the effect of the leakage term does

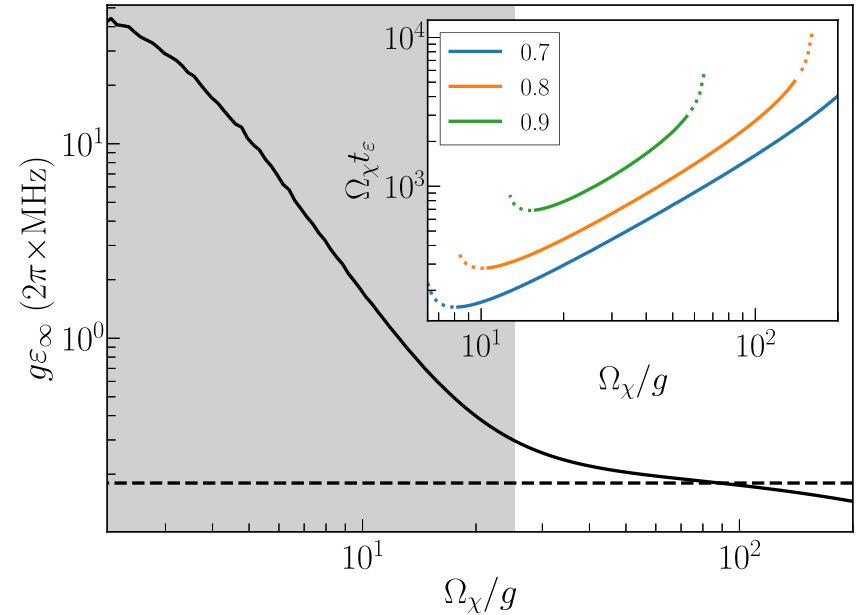

FIG. 5. Scaling of steady-state error for even-parity Bell state preparation, calculated via master equation simulations including the counter-rotating terms, with $\chi_{1,2}=2 \pi \times 10 \mathrm{MHz}, T_{1}=$ $100 \mu \mathrm{s}$, and $T_{2}=200 \mu \mathrm{s}$. The gray region corresponds to the parameter regime where counter-rotating terms induce a fidelity vs. speed tradeoff. The downturn of the curve in the region of large $\Omega_{\chi} / g$ is due to $g$ becoming comparable to $\gamma_{1}$. The dashed line the steady-state error calculated with Eq. (13). (Inset) Time $t_{\varepsilon}$ taken to reach a given fidelity threshold, for the same parameters. When the threshold is near the maximum fidelity for a given set of parameters, it will be crossed at a point where the static and dynamical errors are comparable. This changes the scaling of $t_{\varepsilon}$ with $g$, as indicated by the dotted portions of the curves where $g \gg g^{\text {opt }}$ or $g \lesssim \gamma$.

not saturate with increasing $g$. Therefore, in contrast to the resonant case where it is only necessary to optimize $g_{r}$ and $\kappa$, introduction of the leakage necessitates setting the qubitqubit coupling strength $g$ to some optimal value $g^{\text {opt }}$ as well, found numerically for a given $\Omega_{\chi}$. Thus the parameter space can now be split into two qualitatively different regimes of operation depending on the ratio $g^{\text {opt }} / g$. When $g^{\text {opt }} / g \gg 1$ the leakage term has little effect and the scaling behavior of the resonant Hamiltonian is recovered, where $\varepsilon_{\infty}$ and $\tau$ both scale as $1 / g$ assuming $g_{r}$ and $\kappa$ scale optimally with $g$. When $g^{\text {opt }} / g \ll 1$, the achievable steady-state fidelity from the resonant Hamiltonian saturates and the scaling of $\varepsilon_{\infty}$ is dominated by the increasingly strong leakage term. In this regime, the ratio $\varepsilon / \tau$ is no longer constant. These two regimes are demarcated in Fig. 5.

Though $\chi$-mediated leakage constitutes an additional source of error inherent to the approach of using numberselective parametric driving, detailed numerical simulations show that the effect of the resultant off-resonant terms can be mitigated and fidelity can be recovered, either by employing sufficiently large dispersive shifts as shown in Fig. 5 or by the application of optimal control techniques. We plan to study this in detail in a future work.

\section{CONCLUSIONS}

In summary, we have proposed a new paradigm for dissipation engineering that relies on strong parametric coupling working in conjunction with dispersive interactions. This allows the realization of number-selective parametric driving, 
which we employ here to illustrate the scope of this framework by constructing a novel Bell-state stabilization protocol with no inherent tradeoff between fidelity and stabilization rate. This property is enabled by the interplay of both dispersive and parametric interactions, that allow realization of a target state which is a dark state of both the engineered collapse operator and the drive Hamiltonian. In stark contrast to the usual dissipative state preparation protocols-where parasitic couplings out of the target state impose a hierarchy of coupling rates, and low error can be achieved only by driving the system slower than the effective linewidth of the dressed states to maintain resonant driving [33,34]—our scheme allows parametric drive strengths comparable to decay linewidths permitting a simultaneous optimization of decaydominated and coupling-dominated error channels.

Further, the modular nature of this framework suggests natural extensions to multipartite entanglement preparation. Such considerations become increasingly crucial as dissipative engineering protocols are extended to larger quantum systems, where the speed of state transfer needs to beat the decoherence rate due to correlated noise [35]. Parametrically engineered dissipation also opens possibilities for dissipationmediated quantum control in the stabilized state space, by exploiting the phase and amplitude tunability of the target state dynamics.

Additionally, our scheme offers distinct operational advantages over the previously considered schemes for entanglement stabilization in circuit-QED-like platforms. Since there is no direct driving of the bath resonator, the size of excitations in the resonator, $\bar{n}=\left\langle a^{\dagger} a\right\rangle$, remains small. This is a favorable situation from the point of view of avoiding measurement- induced dephasing of the qubits due to photon number fluctuations, which grows as $\Gamma_{m} \sim \bar{n} \kappa$ [36]. Further, our scheme employs no photon-selectivity for shuttling excitations across the resonator ladder unlike the usual dispersively coupled schemes [31]. Driving number-selective transitions usually places stringent requirements on matching the dispersive shifts, $|\Delta \chi| \ll \chi^{2} / \kappa \sqrt{\bar{n}}$, which ceases to be a constraint for parametrically driven qubit-resonator transitions. Finally, since our scheme has no direct qubit driving, there are no associated Stark shifts and thus no power-dependent pump detuning is necessary.

\section{ACKNOWLEDGMENTS}

We thank Christian Kraglund Andersen, Luke Govia, and Lorenza Viola for helpful discussions, and Philipp Miotti for careful reading of the manuscript. This research was supported by the Department of Energy under grant DE-SC0019461. F.R. acknowledges financial support by a Feodor-Lynen fellowship from the Alexander von Humboldt Foundation.

\section{APPENDIX A: EFFECTIVE HAMILTONIAN DERIVATION}

In order to construct the effective Hamiltonians introduced in Eqs. (5) and (6), we first perform a Schrieffer-Wolff transformation on the dispersive part of the Hamiltonian to diagonalize the dispersive coupling between the qubits and resonator. Using the convention $Z|g\rangle=+|g\rangle$ and $Z|e\rangle=-|e\rangle$, the Hamiltonian becomes

$$
\begin{aligned}
\tilde{\mathcal{H}}= & \frac{\omega_{1}+2 \chi_{1} a^{\dagger} a}{2} Z_{1}+\frac{\omega_{2}+2 \chi_{2} a^{\dagger} a}{2} Z_{2}+\omega_{r} a^{\dagger} a+\left(\tilde{g}_{12}(t)-\frac{\bar{g}_{1 r} \bar{g}_{2 r}}{\Delta_{1 r}}-\frac{\bar{g}_{1 r} \bar{g}_{2 r}}{\Delta_{2 r}}\right) X_{1} X_{2} \\
& +\left(g_{1 r}^{+} e^{i\left(\omega_{1 r}^{+} t+\phi_{1 r}^{+}\right)}+g_{1 r}^{-} e^{i\left(\omega_{1 r}^{-} t+\phi_{1 r}^{-}\right)}+\text {c.c. }\right) X_{1} X_{r}+\left(g_{2 r}^{+} e^{i\left(\omega_{2 r}^{+} t+\phi_{2 r}^{+}\right)}+g_{2 r}^{-} e^{i\left(\omega_{2 r}^{-} t+\phi_{2 r}^{-}\right)}+\text {c.c. }\right) X_{2} X_{r},
\end{aligned}
$$

with dispersive shifts $\chi_{j} \equiv \bar{g}_{j r}^{2} / \Delta_{j r}$ where $\Delta_{j r} \equiv \omega_{r}-\omega_{j}$. We then move into the interaction frame defined by the bare cavity frequency and dressed qubit frequencies via the unitary $U=U_{f} U_{1} U_{2}$ with commuting factors

$$
\begin{aligned}
U_{f} & =\exp \left[i\left(\frac{\omega_{1}}{2} Z_{1}+\frac{\omega_{2}}{2} Z_{2}+\omega_{r} a^{\dagger} a\right) t\right], \\
U_{1} & =\exp \left[i \chi_{1} a^{\dagger} a Z_{1} t\right], \\
U_{2} & =\exp \left[i \chi_{2} a^{\dagger} a Z_{2} t\right] .
\end{aligned}
$$

In this interaction frame, Eq. (A1) becomes

$$
\begin{aligned}
\tilde{\mathcal{H}}_{I}= & \left(g_{12}^{+} e^{i\left(\omega_{12}^{+} t+\phi_{12}^{+}\right)}+g_{12}^{-} e^{i\left(\omega_{12}^{-} t+\phi_{12}^{-}\right)}-\frac{\bar{g}_{1 r} \bar{g}_{2 r}}{\Delta_{1 r}}-\frac{\bar{g}_{1 r} \bar{g}_{2 r}}{\Delta_{2 r}}\right) \\
& \times \sum_{n=0}^{\infty}\left[e^{-i\left[\omega_{1}+\omega_{2}-2 n\left(\chi_{1}+\chi_{2}\right)\right] t}|g, g, n\rangle\left\langle e, e, n\left|+e^{-i\left[\omega_{1}-\omega_{2}-2 n\left(\chi_{1}-\chi_{2}\right)\right] t}\right| g, e, n\right\rangle\langle e, g, n|\right] \\
& +\left(g_{1 r}^{+} e^{i\left(\omega_{1 r}^{+} t+\phi_{1 r}^{+}\right)}+g_{1 r}^{-} e^{i\left(\omega_{1 r}^{-} t+\phi_{1 r}^{-}\right)}\right) \\
& \times \sum_{n=0}^{\infty} \sum_{q_{2} \in\{g, e\}} \sqrt{n+1}\left[e^{-i\left[\omega_{1}+\omega_{r}-\chi_{1}(2 n+1)+\chi_{2}\left\langle q_{2}\left|Z_{2}\right| q_{2}\right\rangle\right] t}\left|g, q_{2}, n\right\rangle\left\langle e, q_{2}, n+1\right|\right. \\
& \left.\quad+e^{-i\left[\omega_{1}-\omega_{r}-\chi_{1}(2 n+1)-\chi_{2}\left\langle q_{2}\left|Z_{2}\right| q_{2}\right\rangle\right] t}\left|g, q_{2}, n+1\right\rangle\left\langle e, q_{2}, n\right|\right]
\end{aligned}
$$




$$
\begin{aligned}
&+\left(g_{2 r}^{+} e^{i\left(\omega_{2 r}^{+} t+\phi_{2 r}^{+}\right)}+g_{2 r}^{-} e^{i\left(\omega_{2 r}^{-} t+\phi_{2 r}^{-}\right)}\right) \\
& \times \sum_{n=0}^{\infty} \sum_{q_{1} \in\{g, e\}} \sqrt{n+1}\left[e^{-i\left[\omega_{2}+\omega_{r}-\chi_{2}(2 n+1)+\chi_{1}\left\langle q_{1}\left|Z_{1}\right| q_{1}\right\rangle\right] t}\left|q_{1}, g, n\right\rangle\left\langle q_{1}, e, n+1\right|\right. \\
&\left.+e^{-i\left[\omega_{2}-\omega_{r}-\chi_{2}(2 n+1)-\chi_{1}\left\langle q_{1}\left|Z_{1}\right| q_{1}\right\rangle\right] t}\left|q_{1}, g, n+1\right\rangle\left\langle q_{1}, e, n\right|\right]+ \text { H.c. }
\end{aligned}
$$

where we have written the Hamiltonian in Dirac notation using the basis states $\left|q_{1}, q_{2}, n\right\rangle \equiv\left|q_{1}\right\rangle \otimes\left|q_{2}\right\rangle \otimes|n\rangle$ defined by the qubit polarizations $q_{1,2}$ and the resonator excitation number $n$.

Section II states the frequencies necessary to target any maximally entangled state of a fixed parity. Using the frequencies required to stabilize an odd-parity state, the Hamiltonian becomes

$$
\begin{aligned}
\widetilde{\mathcal{H}}_{I}= & g_{12}^{+} e^{i \phi_{12}^{+}}\left[|g, g, 0\rangle\left\langle e, e, 0\left|+e^{2 i\left(\chi_{1}+\chi_{2}\right) t}\right| g, g, 1\right\rangle\langle e, e, 1|\right] \\
& +g_{12}^{-} e^{i \phi_{12}^{-}}\left[e^{-2 i\left(\chi_{1}-\chi_{2}\right) t}|g, e, 0\rangle\langle e, g, 0|+| g, e, 1\rangle\left\langle e, g, 1\left|+e^{+2 i\left(\chi_{1}-\chi_{2}\right) t}\right| g, e, 2\right\rangle\langle e, g, 2|\right] \\
& +g_{2 r}^{+} e^{i \phi_{2 r}^{+}}\left[|g, g, 0\rangle\langle g, e, 1|+| e, g, 0\rangle\left\langle e, e, 1\left|+e^{-2 i \chi_{1} t}\right| g, g, 0\right\rangle\left\langle g, e, 1\left|+e^{+2 i \chi_{1} t}\right| e, g, 0\right\rangle\langle e, e, 1|\right] \\
& +g_{1 r}^{+} e^{i \phi_{1 r}^{+}}\left[|g, g, 0\rangle\langle e, g, 1|+| g, e, 0\rangle\left\langle e, e, 1\left|+e^{-2 i \chi_{2} t}\right| g, g, 0\right\rangle\left\langle e, g, 1\left|+e^{+2 i \chi_{2} t}\right| g, e, 0\right\rangle\langle e, e, 1|\right]+\text { H.c. },
\end{aligned}
$$

where we have kept only the static and the dominant counter-rotating terms. The static part of this Hamiltonian, with the resonator truncated to its lowest two energy levels, gives the effective Hamiltonian, stated earlier in Eq. (6),

$$
\mathcal{H}_{\mathrm{eff}}^{\text {odd }}=g_{12}^{+} e^{i \phi_{12}^{+}}\left(1-\left\langle a^{\dagger} a\right\rangle\right) \sigma_{1} \sigma_{2}+g_{12}^{-} e^{i \phi_{12}^{-}}\left\langle a^{\dagger} a\right\rangle \sigma_{1} \sigma_{2}^{\dagger}+\left(g_{1 r}^{+} e^{i \phi_{1 r}^{+}} \sigma_{1}^{\dagger}+g_{2 r}^{+} e^{i \phi_{2 r}^{+}} \sigma_{2}^{\dagger}\right) a^{\dagger}+\text { H.c. },
$$

where we have again expressed the Hamiltonian in terms of canonical operators in this truncated space.

\section{APPENDIX B: PARAMETRIC COUPLER DESIGN}

In our proposed circuit-QED implementation [Fig. 1(c)], the tunable parametric coupling is realized through modulation of the SQUID inductance with an external flux line according to $L_{\mathrm{sq}}=\Phi_{0} /\left(2 \pi I_{c} \cos \left(\pi \Phi / \Phi_{0}\right)\right)$, where $I_{c}$ is the critical current of the Josephson junctions, $\Phi$ is the flux threading the squid loop and $\Phi_{0}$ is the magnetic flux quantum. This leads to a static coupling rate $g_{j r}$ between the qubit $j$ and the resonator as [21,37]

$$
g_{j r}(\Phi) \approx \frac{L_{\mathrm{sq}}(\Phi)}{2 \sqrt{L_{r} L_{j}}} \sqrt{\omega_{j} \omega_{r}}
$$

where $L_{j}$ and $\omega_{j}$ denote the inductance and plasma frequency of the $j$ th transmon, respectively, while $L_{r}$ and $\omega_{r}$ denote the inductance and frequency of the resonator, respectively. We see that the coupling rate is proportional to the participation ratio between the SQUID and qubit/resonator inductance. For small flux modulation amplitudes, the parametric coupling rate resulting from a flux drive $\Phi(t)=\delta \Phi_{j r} \cos \left(\omega_{p j} t+\phi_{p j}\right)$ is given by $g_{j r}=\left(\partial g_{j r} / \partial \Phi\right) \delta \Phi_{j r}$. Figure 6(a) shows a simulation of the qubit-qubit and qubit-resonator coupling rates as a function of flux, for a typical set of circuit-QED parameters. The static coupling rates can be easily tuned over more than $100 \mathrm{MHz}$, for a flux modulation within half-a-period of flux quantum. In the coupler design presented here, $L_{\mathrm{sq}} \ll L_{j}$ to protect the qubits against flux noise from the SQUID. Further, in this architecture the self-resonance frequency of the coupler can be designed to be much higher $(\sim 20-40 \mathrm{GHz})$ than qubit and resonator frequencies of interest for typical specific capacitances associated with aluminum films.

\section{Coupler-induced qubit decoherence}

For the theoretical results presented in this paper, we considered an ideal parametric coupler which induces no additional decay channel on the qubit subsystem. Here we present some empirical estimates of qubit decoherence induced due to coupler architecture presented in Fig. 1(c). We first estimate qubit relaxation due to modification of the effective input impedance, seen by the qubit circuit, by the input flux line. The resultant lifetime $T_{1 j}$ of qubit $j$ limited by coupler impedance can be expressed as

$$
T_{1 j}=\frac{L_{j}}{\Re\left\{Z_{\text {in }}\left[\omega_{j}\right]\right\}},
$$

where $\mathfrak{R}\left\{Z_{\text {in }}\left[\omega_{j}\right]\right\}$ is the environment impedance seen by the qubit $j$ at its resonant frequency. As a first approximation, we can assume that the flux line is a transmission line with characteristic impedance $Z_{0}=50 \Omega$, that terminates into an inductance $L_{0}$ sharing a mutual inductance $M$ with the SQUID loop. We can then compute $Z_{\text {in }}$ as

$$
Z_{\text {in }}[\omega]=i \omega\left(L_{\mathrm{sq}}-M\right)+\frac{i \omega M\left(i \omega\left(L_{0}-M\right)+Z_{0}\right)}{i \omega L_{0}+Z_{0}},
$$

where $L_{\mathrm{sq}}$ denotes the inductance of the SQUID loop. Therefore the qubit lifetime in the limit $L_{j} \gg L_{0}, L_{\mathrm{sq}}$ is

$$
T_{1 j}=\frac{L_{j}\left(\omega_{j}^{2} L_{0}^{2}+Z_{0}^{2}\right)}{\omega_{0}^{2} M^{2} Z_{0}} .
$$

For typical values of the parameters in circuit-QED parameters, $L_{j}=20 \mathrm{nH}, \omega_{j}=2 \pi \times 5 \mathrm{GHz}, L_{0}=0.1 \mathrm{nH}, M=$ $2 \mathrm{pH}$, we obtain the estimated $T_{1 j} \approx 240 \mu \mathrm{s}$. In general we expect that the environment impedance might be complex and modified by resonances in the control line, which could further limit qubit lifetime via Purcell decay. This can be mitigated by placing a notch filter centered at the qubit 

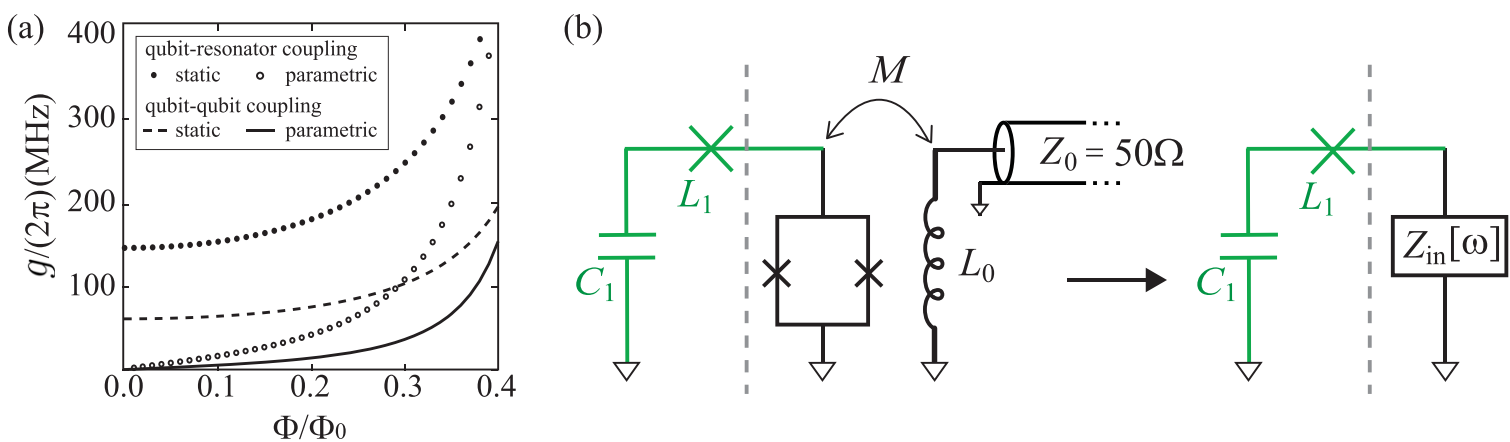

FIG. 6. (a) Simulation results for qubit-resonator and qubit-qubit static and parametric coupling rates, as a function of the flux through the SQUID loop for the circuit introduced in Fig. 1(c). The simulation parameters were $E_{J 1} / E_{C 1}=20, E_{J 2} / E_{C 2}=30, L_{r}=5 \mathrm{nH}$, $\omega_{1}=2 \pi \times 4 \mathrm{GHz}, \omega_{2}=2 \pi \times 6 \mathrm{GHz}, \omega_{r}=2 \pi \times 10 \mathrm{GHz}$, and $\delta \Phi=0.1 \Phi_{0}$. (b) Circuit schematic showing the inductive coupling of the qubit to the flux line, mediated through the coupler, and series LCR circuit obtained using the effective input impedance seen by the qubit due to the flux coupling line.

frequencies into the coupler flux line [21]. Designing such a filter should be straightforward as long as the frequencies of the parametric pumps are well separated from the qubit and resonator frequencies.

The flux noise seen by the coupler can also lead to frequency jitter in the qubit, leading to additional dephasing. To estimate this, we consider $1 / f$-type flux noise spectrum

$$
S_{\Phi \Phi}\left[\omega_{j}\right]=\frac{A^{2}}{\omega_{j}}(2 \pi \times 1 \mathrm{~Hz}),
$$

for which the dephasing time can be estimated as $T_{2 j}^{-1} \approx \Delta \omega_{j}$, where

$$
\begin{aligned}
\omega_{j} & =\left|\frac{\partial \omega_{j}}{\partial \Phi}\right| \Delta \Phi \\
& =\frac{\omega_{j}}{2\left(L_{j}+L_{\mathrm{sq}}(\Phi)\right)}\left(\frac{\partial L_{\mathrm{sq}}(\Phi)}{\partial \Phi}\right) \Delta \Phi \\
& \approx\left(\frac{\pi \omega_{j}}{2}\right) \frac{L_{\mathrm{sq}}(\Phi)}{L_{j}+L_{\mathrm{sq}}(\Phi)} \tan \left(\frac{\pi \Phi}{\Phi_{0}}\right) \sec \left(\frac{\pi \Phi}{\Phi_{0}}\right) \Delta \Phi,
\end{aligned}
$$

with average flux fluctuation

$$
\Delta \Phi=\left(\int_{\omega_{\min }}^{\omega_{\max }} d \omega S_{\Phi \Phi}[\omega]\right)^{1 / 2} .
$$

The above expression is logarithmic in the upper and lower cutoff frequencies, with the latter typically set by the measurement bandwidth and ensuring the convergence of the integral [38]. For typical flux noise amplitudes, $A=1-2 \mu \Phi_{0}$, and circuit parameters [see Fig. 6(a)], this gives an estimated $T_{2 j}^{*} \geqslant 50 \mu \mathrm{s}$. A gradiometric design for the coupler can be used to further insulate the junction from the flux noise due to coupling inductance.

\section{APPENDIX C: JUSTIFICATION FOR THE MASTER EQUATION}

Having constructed an effective Hamiltonian describing the evolution of the system and bath, we now outline the justification behind the full master equation presented as Eq. (8). Since $\kappa \gg \gamma_{1,2}$, our analysis will quantify the effect of the dominant dissipation channel (resonator decay) on the system of two qubits in the presence of strong parametric coupling between the qubits and the resonator. To this end, considering a bosonic environment for the resonator we can write

$$
\begin{aligned}
\mathcal{H}_{S}= & \frac{\omega_{1}}{2} Z_{1}+\frac{\omega_{2}}{2} Z_{2}+\omega_{r} a^{\dagger} a \\
& +\tilde{g}_{12}(t) X_{1} X_{2}+\tilde{g}_{1 r}(t) X_{1} X_{r}+\tilde{g}_{2 r}(t) X_{2} X_{r}, \\
\mathcal{H}_{E}= & \sum_{\alpha} v_{\alpha} r_{\alpha}^{\dagger} r_{\alpha}, \\
\mathcal{H}_{S E}= & \sum_{\alpha} \mu_{\alpha}^{r} r_{\alpha}^{\dagger} a+\text { H.c. }
\end{aligned}
$$

We first diagonalize the static dispersive coupling by performing a Schrieffer-Wolff Transformation. To leading order in the parameter $g_{j r} / \Delta_{j r}$, this transforms the system-environment Hamiltonian as

$$
\tilde{\mathcal{H}}_{S E}=\sum_{\alpha} \mu_{\alpha}^{r} r_{\alpha}^{\dagger}\left(a-\sum_{j=1,2} \frac{\bar{g}_{j r}(t)}{\Delta_{j r}} \sigma_{j}\right)+\text { H.c. }
$$

Next, moving into a rotating frame for both the system and environment gives,

$$
\begin{aligned}
\tilde{\mathcal{H}}_{S E, I}= & \sum_{\alpha} \mu_{\alpha}^{r} r_{\alpha}^{\dagger} a e^{i\left(v_{\alpha}-\omega_{r}-2 \sum_{j=1,2} \chi_{j}\left\langle\sigma_{j}^{Z}\right\rangle\right) t} \\
& -\sum_{\alpha} \sum_{j=1,2} \mu_{\alpha}^{r} \frac{\bar{g}_{j r}}{\Delta_{j r}} r_{\alpha}^{\dagger} \sigma_{j} e^{i\left(v_{\alpha}-\omega_{j}-2\left\langle a^{\dagger} a\right\rangle \chi_{j}\right) t},
\end{aligned}
$$

with qubit frequencies incorporating the corresponding static dispersive shifts alone, $\chi_{j}=\bar{g}_{j r}^{2} / \Delta_{j r}$. Reintroducing the parametric interaction as a time-dependent perturbation on the mixing coefficient, the above interaction can be rewritten in the form

$$
\tilde{\mathcal{H}}_{S E, I}=R(t) \Gamma^{\dagger}(t)+\sum_{j=1,2} Q_{j}(t) \Gamma^{\dagger}(t)+\text { H.c. }
$$


where

$$
\begin{aligned}
& R(t)=a e^{-i \omega_{r}^{\prime} t} \\
& Q(t)=\left(\frac{\tilde{g}_{j r}(t)}{\Delta_{j r}}\right) \sigma_{j} e^{-i \omega_{j}^{\prime} t} \\
& \Gamma(t)=\sum_{\alpha} \mu_{\alpha}^{r *} r_{\alpha} e^{-i v_{\alpha} t} .
\end{aligned}
$$

with $\omega_{r}^{\prime}=\omega_{r}+2 \sum_{j=1,2}\left\langle\sigma_{j}^{Z}\right\rangle \chi_{j}$ and $\omega_{j}^{\prime}=\omega_{j}+2\left\langle a^{\dagger} a\right\rangle \chi_{j}$. Note that we have ignored the inertial term that arises if one performs a full time-dependent Schrieffer-Wolff transformation which leads to additional dispersive shifts due to parametric interactions between the qubits and resonator; this approximation is valid in the RWA regime [39], $\left|g_{j r}\right| \ll \chi<$ $\bar{g}_{j r} \ll \Delta_{j r}$ (white region in Fig. 5), required to maintain the concurrent scaling of target fidelity and stabilization rate.

Assuming Markovian evolution and taking the zerotemperature limit for the environment [40], leads to the following terms in the master equation:

$$
\begin{aligned}
\dot{\rho}_{I}(t)= & \left(a \rho_{I}(t) a^{\dagger}-a^{\dagger} a \rho_{I}(t)\right) \int_{0}^{t} d t^{\prime} e^{-i \omega_{r}^{\prime}\left(t^{\prime}-t\right)}\left\langle\Gamma(t) \Gamma^{\dagger}\left(t^{\prime}\right)\right\rangle \\
& +\sum_{j=1,2}\left(\frac{\bar{g}_{j r}}{\Delta_{j r}}\right)^{2}\left(\sigma_{j} \rho_{I}(t) \sigma_{j}^{\dagger}-\sigma_{j}^{\dagger} \sigma_{j} \rho_{I}(t)\right) \\
& \times \int_{0}^{t} d t^{\prime} e^{-i \omega_{j}^{\prime}\left(t^{\prime}-t\right)}\left\langle\Gamma(t) \Gamma^{\dagger}\left(t^{\prime}\right)\right\rangle \\
& +\sum_{j=1,2}\left|\frac{g_{j r}}{\Delta_{j r}}\right|^{2}\left(\sigma_{j} \rho_{I}(t) \sigma_{j}^{\dagger}-\sigma_{j}^{\dagger} \sigma_{j} \rho_{I}(t)\right) \\
& \times \int_{0}^{t} d t^{\prime}\left(e^{-i\left(\omega_{j}^{\prime}+\omega_{j r}\right)\left(t^{\prime}-t\right)}+e^{-i\left(\omega_{j}^{\prime}-\omega_{j r}\right)\left(t^{\prime}-t\right)}\right)\left\langle\Gamma(t) \Gamma^{\dagger}\left(t^{\prime}\right)\right\rangle \\
& + \text { H.c. } \quad \text { (C6) }
\end{aligned}
$$

Here we have decomposed the qubit-resonator interaction into two parts, one corresponding to the static qubit-resonator coupling and the other due to the time-varying parametric part $g_{j r}(t)=g_{j r} e^{i\left(\omega_{j r} t+\phi_{j r}\right)}+$ H.c. Introducing a continuous spectral density of states for the environment,

$$
\left\langle\Gamma(t) \Gamma^{\dagger}\left(t^{\prime}\right)\right\rangle=\int_{0}^{\infty} d v e^{-i v\left(t-t^{\prime}\right)} \rho(v)|\kappa(v)|^{2},
$$

and using it in Eq. (C6), we obtain

$$
\begin{aligned}
\dot{\rho}_{I}(t)= & -i\left[\Delta, \rho_{I}(t)\right]+\kappa \mathcal{D}[a] \rho_{I}(t) \\
& +\sum_{j=1,2} \kappa\left(\frac{\bar{g}_{j r}}{\Delta_{j r}}\right)^{2} \mathcal{D}\left[\sigma_{j}\right] \rho_{I}(t) \\
& +\sum_{j=1,2} \kappa^{\prime}\left|\frac{g_{j r}}{\Delta_{j r}}\right|^{2} \mathcal{D}\left[\sigma_{j}\right] \rho_{I}(t) .
\end{aligned}
$$

Here we have used

$$
\int_{0}^{t} d t^{\prime} e^{-i(\omega-v)\left(t-t^{\prime}\right)}=\pi \delta(\omega-v)+i P\left(\frac{1}{\omega-v}\right),
$$

with the corresponding decay rates

$$
\begin{aligned}
\kappa(\omega) & =2 \pi \rho(\omega)\left|\mu^{r}(\omega)\right|^{2}, \\
\kappa^{\prime}(\omega) & =2 \pi \rho\left(\omega \pm \omega_{p}\right)\left|\mu^{r}\left(\omega \pm \omega_{p}\right)\right|^{2},
\end{aligned}
$$

and Lamb shift (usually absorbed by redefining bare resonance frequencies)

$$
\Delta=\frac{P}{2 \pi} \int_{0}^{\infty} d \nu \frac{\kappa(\omega)}{\omega-\nu} .
$$

If the decay rate is calculated for the resonator, the corresponding density of states needs to be calculated at the resonator frequency $\omega_{r}^{\prime}$, while for the induced decay on the qubit the spectral density of the environment near $\omega_{j}^{\prime}$ is relevant. As is evident from Eq. (C8), the parametric coupling contributes towards qubit decoherence with a term almost identical to that due to the dispersive-coupling induced Purcell decay, except that the relevant environmental modes that contribute are shifted by $\pm \omega_{p}$. Depending on the choice of pump frequencies, only one of the up/down-converted noise terms maybe relevant; for instance, for the qubit-resonator pump frequencies stated in Appendix A, only the $\omega_{j}^{\prime}+\omega_{j r}$ contributes towards parametrically induced spontaneous emission. Further, under the assumption that the environment spectrum is white, $\kappa \approx \kappa^{\prime}$, leading to a simple modification of the Purcell decay now including the contribution due to parametric coupling. Our numerical simulations show that including these nonlocal dissipation channels do not fundamentally alter the scaling behavior of the scheme. Furthermore the primary effect remains the resonator-induced Purcell decay due to static dispersive coupling, which can be mitigated in experiments using a Purcell filter [41].

Doing a similar analysis for the qubit-environment coupling under Schrieffer-Wolff transformation,

$$
\tilde{\mathcal{H}}_{S E}=\sum_{j=1,2} \sum_{\alpha} \mu_{\alpha, j}^{q} q_{\alpha, j}^{\dagger}\left(\sigma_{j}+\frac{\bar{g}_{j r}(t)}{\Delta_{j r}} a Z_{j}\right)+\text { H.c. }
$$

leads to the following damping superoperators:

$$
\sum_{j=1,2} \gamma \mathcal{D}\left[\sigma_{j}\right] \bullet+\gamma\left(\frac{\bar{g}_{j r}}{\Delta_{j r}}\right)^{2} \mathcal{D}\left[a Z_{j}\right] \bullet+\gamma^{\prime}\left|\frac{g_{j r}}{\Delta_{j r}}\right|^{2} \mathcal{D}\left[a Z_{j}\right] \bullet .
$$

As before, the additional dissipators are weighted by a factor proportional to $\left|g_{j r} / \Delta\right| \ll 1$ and the primary difference due to parametric coupling is the frequency at which environmental noise is sampled: while $\gamma$ corresponds to noise at respective resonant frequencies, $\gamma^{\prime}$ is determined by noise at the qubit frequency shifted by $\pm \omega_{p}$.

\section{Qubit and resonator heating}

Usually terms of type $r_{\alpha}^{\dagger} a^{\dagger}, q_{\alpha, j}^{\dagger} \sigma_{j}^{\dagger}$ in the systemenvironment interaction correspond to negative frequency terms in the environment spectrum, which have zero spectral weight and hence do not contribute towards spontaneous emission. However, in the presence of parametric driving, the environmental modes down to $-\omega_{j r}$ are accessible, leading to environment-mediated excitations of the qubit and resonator even at zero temperature. These processes lead to superoperators of the form

$$
\sum_{j=1,2} \kappa^{\prime}\left|\frac{g_{j r}}{\Delta_{j r}}\right|^{2} \mathcal{D}\left[\sigma_{j}^{\dagger}\right] \bullet, \quad \sum_{j=1,2} \gamma^{\prime}\left|\frac{g_{j r}}{\Delta_{j r}}\right|^{2} \mathcal{D}\left[a^{\dagger}\right] \bullet .
$$




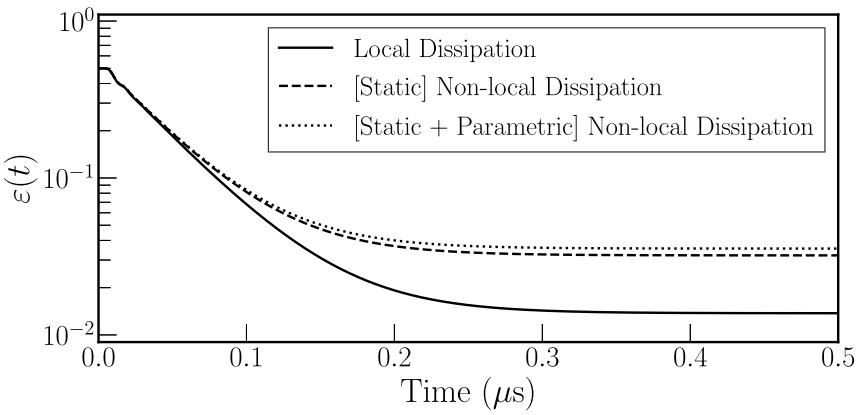

FIG. 7. Time-domain master equation simulations showing the preparation of an even-parity Bell state, with $g=(4 / 3) g_{r}=$ $(2 / 3) \kappa=2 \pi \times 50 \mathrm{MHz}$ and $T_{1}=T_{2}=10 \mu \mathrm{s}$. The solid curve is calculated using Eq. (8), which incorporates only local dissipation. The dashed curve additionally includes the nonlocal dissipation induced by the static couplings alone, with the dominant effect being the Purcell decay of the qubits. The dotted line extends this calculation to include parametrically induced nonlocal dissipation, dominated by qubit heating and additional Purcell decay.

Nonetheless, in the regime of interest, such environmentinduced heating of the system and the effect of the additional damping superoperators due to parametric couplings in Eqs. (C8) and (C13) remain negligible as shown in Fig. 7, and hence are neglected in the analysis presented in Sec. III. We note that in experiments these effects should be smaller still, as our calculations assuming a white noise spectrum overestimate the decay rates.

\section{APPENDIX D: ROBUSTNESS TO IMPERFECTIONS AND ASYMMETRIES}

Numerical simulations show that the scheme is robust to imperfections, with minimal performance loss occurring for as large as $50 \%$ deviations from optimal values of $g_{r}$ or $\kappa$ as shown in Fig. 8(a). The scheme remains similarly robust in the presence of large asymmetries in the qubit-qubit couplings $g_{12}^{+} \neq g_{12}^{-}$. In fact, such an asymmetry can provide slightly improved performance when $g_{12}^{-}>g_{12}^{+}$. However, asymmetries in the qubit-resonator couplings $g_{1 r} \neq g_{2 r}$ have a much larger negative impact on the achievable fidelities, as shown in Fig. 8(b). This is to be expected, as these couplings are used exclusively to engineer the collapse operator acting on the two-qubit subspace. Thus any asymmetry in the coupling strengths changes the form of the engineered system collapse operator in such a way that the target state $|\xi\rangle$ is no longer a dark state. Explicitly, asymmetric qubit-resonator coupling strengths lead to a coherent coupling between the target state $|\xi, 0\rangle$ and $|\bar{C}, 1\rangle$. Since the parametric coupling strengths can be precisely matched through the amplitude of the pumps, in practice this is not a significant limitation.

The effect of qubit detunings $\delta_{1,2}$ on the achievable fidelities is shown in Fig. 8(c). When these detunings are nonzero, there may be spurious coupling introduced between the target state $|\xi\rangle$ and $|\bar{\xi}\rangle$. This coupling is introduced if either $\delta_{1}+$ $\delta_{2} \neq 0$ or $\delta_{1}-\delta_{2} \neq 0$, when stabilizing an even- or odd-parity state, respectively. It is, therefore, natural to parametrize the qubit detunings in terms of the sum and difference $\delta_{1} \pm \delta_{2}$. The protocol is sensitive to the parameter which leads to the spurious coupling, and mostly insensitive to the conjugate parameter. This is again not a limitation of the scheme, given the precise frequency control available to experiments. Further, the scheme is weakly sensitive to resonator detuning, though it demonstrates a slight improvement of steady-state fidelity with some amount of detuning $0<\left|\Delta_{r}\right|<g_{r}$. This is because detuning the resonator reduces the Rabi contrast between the states $|C, 0\rangle$ and $|\bar{\xi}, 1\rangle$, which ultimately leads to a slightly increased stabilization rate and thus steady-state fidelity.
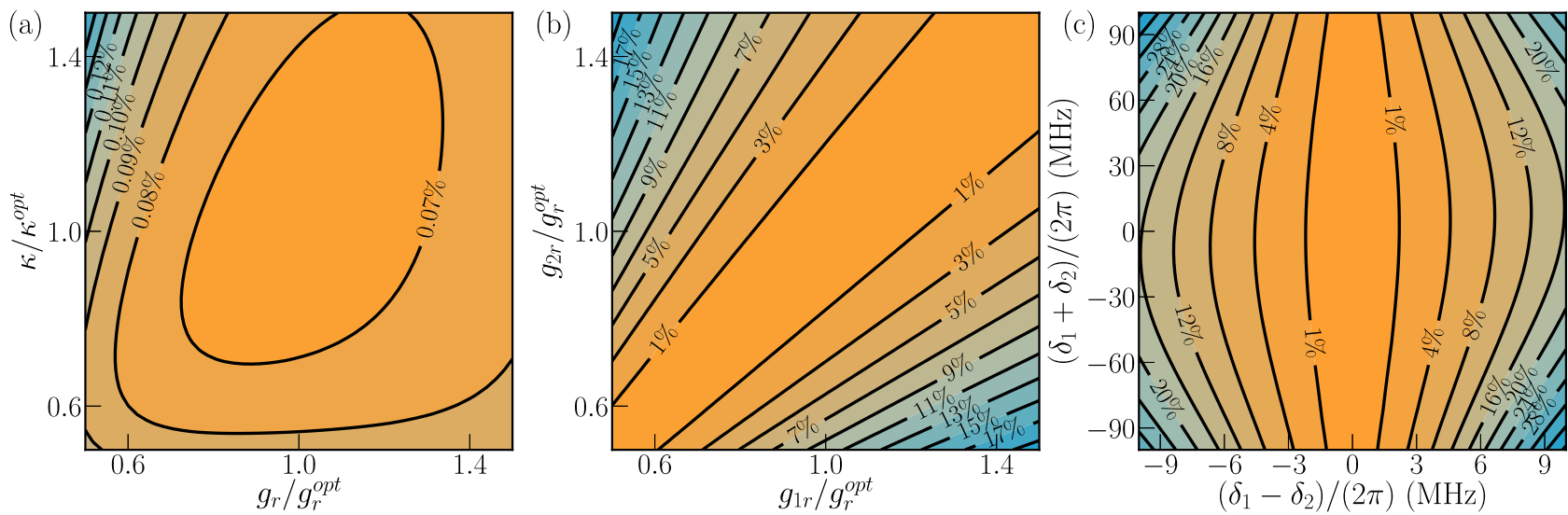

FIG. 8. (a) Steady-state error as a function of the deviation of the resonator linewidth $\kappa$ and qubit-resonator coupling strength $g_{r}$ from their respective optimal values, $\kappa^{\mathrm{opt}}=2 g_{r}^{\mathrm{opt}}=(3 / 2) g$ with $g=2 \pi \times 50 \mathrm{MHz}, T_{1}=100 \mu \mathrm{s}$, and $T_{2}=200 \mu$ s. (b) Steady-state error when preparing $|S\rangle$ with asymmetric qubit-resonator couplings, calculated with the same optimal values and decoherence rates. Asymmetry in the qubit-resonator couplings has a detrimental effect on the achievable fidelity. This can be remedied by leveraging the tunable strength of the parametric couplings. (c) Steady-state error in the presence of qubit detunings, for the same parameter values. When $\delta_{1}-\delta_{2} \neq 0$, there is spurious coupling between $|S\rangle$ and $|T\rangle$ which limits the achievable fidelity. The scheme is mostly insensitive to $\delta_{1}+\delta_{2} \neq 0$ when stabilizing an odd-parity state. When stabilizing an even-parity state, the sum of $\delta_{1}$ and $\delta_{2}$ leads to spurious coupling rather than the difference. 
[1] B. Kraus, H. P. Büchler, S. Diehl, A. Kantian, A. Micheli, and P. Zoller, Phys. Rev. A 78, 042307 (2008).

[2] F. Verstraete, M. M. Wolf, and J. I. Cirac, Nat. Phys. 5, 633 (2009).

[3] M. B. Plenio, S. F. Huelga, A. Beige, and P. L. Knight, Phys. Rev. A 59, 2468 (1999).

[4] F. Benatti, R. Floreanini, and M. Piani, Phys. Rev. Lett. 91, 070402 (2003).

[5] F. Ticozzi and L. Viola, Quantum Inf. Comput. 14, 265 (2014).

[6] F. Reiter, D. Reeb, and A. S. Sørensen, Phys. Rev. Lett. 117, 040501 (2016).

[7] S. Clark, A. Peng, M. Gu, and S. Parkins, Phys. Rev. Lett. 91, 177901 (2003).

[8] A. S. Parkins, E. Solano, and J. I. Cirac, Phys. Rev. Lett. 96, 053602 (2006).

[9] Y. Lin, J. P. Gaebler, F. Reiter, T. R. Tan, R. Bowler, A. S. Sorensen, D. Leibfried, and D. J. Wineland, Nature (London) 504, 415 (2013).

[10] P. Schindler, M. Müller, D. Nigg, J. T. Barreiro, E. A. Martinez, M. Hennrich, T. Monz, S. Diehl, P. Zoller, and R. Blatt, Nat. Phys. 9, 361 (2013).

[11] D. Kienzler, H.-Y. Lo, B. Keitch, L. de Clercq, F. Leupold, F. Lindenfelser, M. Marinelli, V. Negnevitsky, and J. P. Home, Science 347, 53 (2015).

[12] S. Shankar, M. Hatridge, Z. Leghtas, K. M. Sliwa, A. Narla, U. Vool, S. M. Girvin, L. Frunzio, M. Mirrahimi, and M. H. Devoret, Nature (London) 504, 419 (2013).

[13] M. E. Kimchi-Schwartz, L. Martin, E. Flurin, C. Aron, M. Kulkarni, H. E. Tureci, and I. Siddiqi, Phys. Rev. Lett. 116, 240503 (2016).

[14] A. W. Carr and M. Saffman, Phys. Rev. Lett. 111, 033607 (2013).

[15] D. D. Bhaktavatsala Rao and K. Mølmer, Phys. Rev. Lett. 111, 033606 (2013).

[16] H. Krauter, C. A. Muschik, K. Jensen, W. Wasilewski, J. M. Petersen, J. I. Cirac, and E. S. Polzik, Phys. Rev. Lett. 107, 080503 (2011).

[17] P.-B. Li, S.-Y. Gao, H.-R. Li, S.-L. Ma, and F.-L. Li, Phys. Rev. A 85, 042306 (2012).

[18] M. S. Allman, F. Altomare, J. D. Whittaker, K. Cicak, D. Li, A. Sirois, J. Strong, J. D. Teufel, and R. W. Simmonds, Phys. Rev. Lett. 104, 177004 (2010)

[19] D. C. McKay, S. Filipp, A. Mezzacapo, E. Magesan, J. M. Chow, and J. M. Gambetta, Phys. Rev. Appl. 6, 064007 (2016).

[20] M. Reagor, C. B. Osborn, N. Tezak, A. Staley, G. Prawiroatmodjo, M. Scheer, N. Alidoust, E. A. Sete, N. Didier, M. P. da Silva et al., Sci. Adv. 4, eaao3603 (2018).
[21] Y. Lu, S. Chakram, N. Leung, N. Earnest, R. K. Naik, Z. Huang, P. Groszkowski, E. Kapit, J. Koch, and D. I. Schuster, Phys. Rev. Lett. 119, 150502 (2017).

[22] A. Kamal, J. Clarke, and M. H. Devoret, Nat. Phys. 7, 311 (2011).

[23] P. Roushan, C. Neill, A. Megrant, Y. Chen, R. Babbush, R. Barends, B. Campbell, Z. Chen, B. Chiaro, A. Dunsworth et al., Nat. Phys. 13, 146 (2016).

[24] M. Mirrahimi, Z. Leghtas, V. V. Albert, S. Touzard, R. J. Schoelkopf, L. Jiang, and M. H. Devoret, New J. Phys. 16, 045014 (2014).

[25] S. Puri, C. K. Andersen, A. L. Grimsmo, and A. Blais, Nat. Commun. 8, 15785 (2017).

[26] A. Blais, R.-S. Huang, A. Wallraff, S. M. Girvin, and R. J. Schoelkopf, Phys. Rev. A 69, 062320 (2004).

[27] D. I. Schuster, A. A. Houck, J. A. Schreier, A. Wallraff, J. M. Gambetta, A. Blais, L. Frunzio, J. Majer, B. Johnson, M. H. Devoret, S. M. Girvin, and R. J. Schoelkopf, Nature (London) 445, 515 (2007).

[28] J. Casanova, G. Romero, I. Lizuain, J. J. García-Ripoll, and E. Solano, Phys. Rev. Lett. 105, 263603 (2010).

[29] A. Levy and R. Kosloff, Europhys. Lett. 107, 20004 (2014).

[30] F. Beaudoin, J. M. Gambetta, and A. Blais, Phys. Rev. A 84, 043832 (2011).

[31] Z. Leghtas, U. Vool, S. Shankar, M. Hatridge, S. M. Girvin, M. H. Devoret, and M. Mirrahimi, Phys. Rev. A 88, 023849 (2013).

[32] F. Reiter, L. Tornberg, G. Johansson, and A. S. Sørensen, Phys Rev. A 88, 032317 (2013).

[33] F. Reiter, M. J. Kastoryano, and A. S. Sørensen, New J. Phys. 14, 053022 (2012).

[34] C. Aron, M. Kulkarni, and H. E. Türeci, Phys. Rev. X 6, 011032 (2016).

[35] A. Zwick, G. A. Álvarez, G. Bensky, and G. Kurizki, New J. Phys. 16, 065021 (2014).

[36] J. Gambetta, A. Blais, D. I. Schuster, A. Wallraff, L. Frunzio, J. Majer, M. H. Devoret, S. M. Girvin, and R. J. Schoelkopf, Phys. Rev. A 74, 042318 (2006).

[37] E. Zakka-Bajjani, F. Nguyen, M. Lee, L. R. Vale, R. W. Simmonds, and J. Aumentado, Nat. Phys. 7, 599 (2011).

[38] G. Ithier, E. Collin, P. Joyez, P. J. Meeson, D. Vion, D. Esteve, F. Chiarello, A. Shnirman, Y. Makhlin, J. Schriefl, and G. Schön, Phys. Rev. B 72, 134519 (2005).

[39] Z. Xiao et al. (unpublished).

[40] H. J. Carmichael, Statistical Methods in Quantum Optics: Master Equations and Fokker-Planck Equations, 2nd ed. (Springer, Berlin, 2002), Vol. 1.

[41] M. D. Reed, B. R. Johnson, A. A. Houck, L. DiCarlo, J. M. Chow, D. I. Schuster, L. Frunzio, and R. J. Schoelkopf, Appl. Phys. Lett. 96, 203110 (2010). 